\title{
Treinta años de Antropología en España. Memoria desde la Gazeta
}

Thirty years of Anthropology in Spain. Report from the Gazeta

Celeste Jiménez de Madariaga

Profesora Titular de Antropología Social. Departamento de Historia I. Universidad de Huelva (España) celeste@uhu.es

\section{Francisco Checa Olmos \\ Profesor Titular de Antropología Social. Departamento de Ciencias Humanas y Sociales. Universidad de Almería (España) fcheca@ual.es}

\section{EDITORIAL}

\author{
30 ANIVERSARIO DE LA GAZETA DE ANTROPOLOGÍA \\ NÚMERO COORDINADO POR FRANCISCO CHECA OLMOS Y CELESTE JIMÉNEZ DE MADARIAGA
}

\begin{abstract}
RESUMEN
Las publicaciones periódicas cumplen una función vital para la comunidad científica, dado que son soporte y vehículo para la expresión, divulgación y el debate. Desde esta premisa, el artículo rememora la trayectoria editorial de las revistas de Antropología en España, cuyos inicios quedan asociados a la institucionalización académica de dicha disciplina en el país. De entre ellas, una de las decanas es Gazeta de Antropología, que en 2012 cumplió 30 años desde su primer número; un momento para celebrar sus logros, pero también para la reflexión y el análisis de por dónde puede/debe ir una revista científica. Las posibilidades que ofrece la red permiten compartir la investigación y el conocimiento mucho más rápido que décadas atrás, incluso de forma más económica. Cuestiones que, sin duda, han de ser consideradas.

\section{ABSTRACT}

Periodical publications play a vital role for the scientific community, as they constitute the medium and vehicle for expression, dissemination, and debate. From this premise, the article recalls the editorial history of anthropology journals in Spain, the beginnings of which are associated with academic institutionalization of the discipline in the country. Among them is one of the deans of the Gazeta de Antropología which in 2012 marked 30 years since its first issue, a time to celebrate their achievements, but also for reflection and analysis of where a scientific journal can / should go. The possibilities offered by the Internet allow research and knowledge to be shared much faster than decades ago, and even more economically. These matters certainly need to be considered.
\end{abstract}

\section{Las revistas de Antropología Social y Cultural en España}

El desarrollo de la Antropología Social y Cultural como disciplina científica en España ha estado muy supeditado a su tardía y lenta implantación en los ámbitos académicos, y a su institucionalización disciplinar. Salvo algunos núcleos muy localizados de una incipiente antropología de carácter folclorista y positivista que, en torno a las últimas décadas del siglo XIX nos legará estudios fundamentalmente sobre expresiones culturales regionalistas, y a los que por razones obvias no nos remontaremos en este artículo, la antropología en España no comienza su andadura hasta bien entrada la mitad del siglo XX. Más aún, será a partir de los años 70 cuando se inicie la institucionalización académica de la antropología, entrando a formar parte como materia de estudio de las universidades españolas, si bien desde entonces su expansión ha sido verdaderamente significativa. 
La misma dilación en la implantación de la antropología en España se refleja en la aparición de la producción científica y en las revistas de la especialización editadas, así como en sus momentos de avance y auge. Las publicaciones periódicas cumplen para las comunidades científicas un papel importante en la expresión, divulgación y debate de los intereses científicos, constituyéndose en órganos de expresión e institucionalización de cada disciplina y su profesión. Si bien antes de la década de 1980 encontramos algunas revistas especializadas de antropología, o incluyendo algunos contenidos de temáticas antropológicas, a veces a modo de cuadernillos con formatos muy rústicos, no será hasta esta fecha cuando se gesten la mayor parte de nuestras revistas, en torno a unos diecisiete títulos. Esta eclosión coincide con tres hechos: en primer lugar, la introducción de la antropología en las universidades españolas, al principio como rama de determinadas titulaciones, después como especialidad, para llegar a consolidarse como licenciaturas -actualmente grado- y doctorados -hoy máster-; en segundo lugar, la aparición de asociaciones e institutos de antropología que pretenden aunar a los profesionales de esta disciplina de las distintas Comunidades Autónomas y lugares que han sido -y son- focos de producción y estudios antropológicos; por último, aunque con una menor incidencia, la incorporación de la antropología entre los intereses de algunos organismos públicos, como museos, centros de investigación y fundaciones. De este modo, la mayoría de las revistas antropológicas que se editan en España han estado -y en muchos casos continúan estando- vinculadas a departamentos de antropología social y cultural de las universidades españolas o a asociaciones de antropólogos.

También es cierto que un elevado número de revistas no ha tenido continuidad, agotándose en unos pocos números. Los principales motivos hay que buscarlos en la falta de recursos económicos, el escaso apoyo institucional y el cansancio de quienes constituían las directivas y comités editoriales, dado que su labor era completamente altruista. Publicar una revista con un mínimo de calidad siempre ha supuesto unos gastos de imprenta que difícilmente puede costearse con suscriptores y ventas, mucho menos en épocas donde el total de antropólogos en España era escaso. Las posibilidades de financiación por parte de entidades públicas -o privadas- eran entonces muy complicadas, pues la insuficiente repercusión de la antropología en el panorama intelectual español llevaba a pensar que las ediciones iban a ser poco viables y/o rentables. Por otra parte, es bastante generalizado que la creación de una revista suele producirse por iniciativa de una persona o grupo muy reducido. Ello supone que su trabajo organizativo y de gestión dependa de la voluntariedad y tenacidad de estas personas; consecuentemente, la continuidad es muy inestable. También, pese a las buenas intenciones, la inexperiencia de los promotores les hacía incurrir en un inadecuado proceso de edición, con una inapropiada estructuración de artículos, insuficientes controles de calidad, imprecisa periodicidad, variabilidad temática, etc.

Como se observa, han sido muchas las dificultades para que estas revistas se mantuvieran hasta la actualidad, y las que lo han logrado, siguen encontrándose con verdaderos obstáculos para cumplir los índices de calidad que los repositorios exigen para otorgar una acreditación internacional, lo que, a la postre supone un mayor grado de prestigio, impacto y difusión de cada revista. O dicho de otro modo, la importancia y valor de las revistas españolas de antropología ha de medirse en desiguales condiciones respecto a otras revistas científicas de diferentes disciplinas, dado el apoyo institucional que unas y otras reciben, en el de la antropología bastante escaso. Sin duda, la escasez de buenas revistas de antropología en España ha sido un escollo para la producción investigadora de nuestros antropólogos.

También es cierto que muchas de las dificultades citadas se están superando con la creación de revistas electrónicas o el paso de las tradicionales al formato digital, a veces compaginando ambos tipos de edición. Las facilidades que proporciona internet ha supuesto un repunte de las revistas de antropología en los últimos años y una mayor concienciación por parte de los editores por cumplir los requisitos de calidad: control de la periodicidad, revisión de artículos por pares, normas de publicación para los autores, etc.

Sin entrar en precedentes anteriores, desde la década de 1940 y hasta la mencionada eclosión de la década de los 80, encontramos cuatro revistas con contenidos de temática antropológica en España, de las que solo una se mantiene hasta la actualidad, la Revista de Dialectología y Tradiciones Populares, siendo, pues, la decana de las publicaciones periódicas españolas dedicadas a la antropología social y cultural. La RDTP fue fundada por Vicente García de Diego en la Sección de Tradiciones Populares del 
Instituto Antonio de Nebrija de Filología Clásica, en el Consejo Superior de Investigaciones Científicas de Madrid, en 1944; la publicación nació con el título de Revista de Tradiciones Populares, modificado en 1945 por el actual.

La Revista Española de Antropología Americana, vinculada al Departamento de Historia de América II (Antropología de América) de la Facultad de Geografía e Historia de la Universidad Complutense de Madrid, aparece con esta denominación en el año 1969. Sin embargo sus antecedentes se remontan unos años antes, cuando en 1952, siendo aún Departamento de Antropología y Etnología de América de la Facultadde Filosofía y Letras, se realizaba un Seminario de Estudios Americanistas, de los que se publicaban Trabajos y Conferencias en forma de cuadernillos monográficos. Al ser concebida a modo de una continuidad de estos Trabajos y Conferencias, el número inicial de la revista, con la denominación actual, consta como volumen IV. La iniciativa de esta publicación partió de Manuel Ballesteros Gaibrois, con José Alcina Franch como encargado de la edición.

Desde el año 1969 hasta la actualidad se publica Cuadernos de Etnología y Etnografía de Navarra, auspiciada por el Departamento de Cultura, Turismo y Relaciones Institucionales del Gobierno de Navarra; es otra de las revistas de mayor continuidad y permanencia, no en vano en 2012 apareció el número 87.

Ya en los inicios de la década de los 70 se crean dos revistas que no tendrán continuidad en el tiempo. Por un lado, en la Universidad Complutense de Madrid, Juan Vicente Palerm funda Cuadernos de Antropología Social y Etnología (1970-1974), que desaparece cuando éste regresa a México. Por otro, en Barcelona, en el Centro de Etnología Peninsular del CSIC, Claudio Esteva Fabregat funda Ethnica. Revista de Antropología (1971-1984), que desaparece con el retiro de su fundador, después de veinte números.

Como vemos, muchas son las iniciativas que han visto truncada su trayectoria, o bien se han visto abocadas a una continuidad y periodicidad inciertas, con altibajos y cambios de denominación y patrocinio. Un ejemplo de ello es Anales del Museo del Pueblo Español, cuya primera publicación se remonta a una fecha tan lejana como es 1935, pero no apareciendo el segundo volumen hasta el año 1988 y que se mantendrá de manera discontinua hasta 1993, contando en total con seis números publicados en diversas fechas. Después pasarán a denominarse Nos-Otros, Anales del Museo Nacional de Antropología, tras la reconversión de dicho museo, publicando nueve volúmenes, uno por año, desde 1994 a 2002. Similar proceso de discontinuidad y cambio de denominación se produce en Anthropologica. Revistas de Antropología Filosófica (1973-1977), que publica en su primera etapa cuatro volúmenes. Desde la presentación pública del Instituto de Antropología de Barcelona, Anthropologica pasó a ser portavoz de los miembros del Instituto con la publicación de un número monográfico (1982); para convertirse posteriormente en Anthropologica: Revista de Etnopsicología y Etnopsiquiquiatría (19872000). Tampoco tuvo continuidad Comentaris d'Antropología Cultural (1979-1984), del Departamento de Antropología Cultural de la Universidad de Barcelona, que se perdió tras seis números, más bien desiguales. En el año 1975 apareció el primer número de la revista Etniker Bizkaia como un modesto boletín interno para los colaboradores del grupo de investigación Etniker Bizkaia, dirigido por José Miguel Barandiarán, y cuya pretensión inicial era recoger los trabajos de campo y las guías metodológicas creadas dentro del proyecto del Atlas Etnográfico de Euskadi. Asimismo, en 1975 nacía Narria. Estudios de artes y costumbres populares, vinculada a la Universidad Autónoma de Madrid y al Museo de Artes y Tradiciones, una publicación periódica con un gran número de volúmenes publicados (hasta ciento veinticuatro en el año 2008) gracias al sufrago íntegro de su creadora y directora, Guadalupe GonzálezHontoria y Allendesalazar.

Como decíamos, la década de 1980 será especialmente prolífera en revistas de antropología, coincidiendo con su institucionalización y con el surgimiento de asociaciones profesionales de antropólogos. En el mismo año 80 ya se publican dos revistas: Etnografía española (1980-1995), con una periodicidad irregular de nueve números, pero con la novedad de ser editada por una entidad política, el Ministerio de Cultura, y Quaderns de l'Institut Catala d'Antropología, que se mantiene desde entonces con una periodicidad anual, pasando en 2003 a versión electrónica. 
El año 1982 fue especialmente importante porque llegaron a crearse hasta cuatro revistas, tres de ellas aún están activas. Este es el caso de Gazeta de Antropología, que en este artículo homenajeamos por su treinta aniversario y que páginas abajo nos extenderemos con más detalles, gracias a la iniciativa de la Asociación Granadina de Antropología. Cuadernos de Sección de Eusko Ikaskuntza-Sociedad de Estudios Vascos, revista de la que aparecen trece números bajo esta denominación, a partir de1997 cambia el título por Zainak. Cuadernos de Antropología-Etnografía. En Tarragona se funda Arxiu d'Etnografia de Catalunya. Revista d'Antropologia Social, en el seno del Departament d'Antropologia Social i Filosofia de la Universitat Rovira i Virgili, si bien tras 10 números estuvo durante un tiempo sin publicarse, se retoma en 2011 con el apoyo del Institut Tarragonès d'Antropologia. La última de las revistas fundadas este año dejó de publicarse tras varios números, Alcaveras. Revista de Antropología (1982-1987), gestada por la Asociación Madrileña de Antropología. Al año siguiente, en 1983, el Instituto Aragonés de Antropología fundaTemas de Antropología Aragonesa, que se ha mantenido hasta la actualidad, ofreciendo su número 18 en el año2012.

En esta década también destacan los años 1987 y 1988 por el elevado número de revistas que aparecen. Antropologies (1987-1997) sale a la luz desde la Associasió Anthropologies de la Facultat de Geografia i Història de la Universitat de Barcelona. La revista Anales de la Fundación Joaquín Costa (1987-), aunque con contenidos no exclusivamente antropológicos, ha tenido siempre un apartado dedicado a la antropología social, fruto de los encuentros anuales que organiza el profesor D. Carmelo Lisón; hasta el año 2011 tiene publicados veintiséis números.

En Sevilla, auspiciada por la Fundación Machado y dirigida por Salvador Rodríguez Becerra, aparece El Folk-Lore Andaluz. Revista de Cultura Tradicional. $2^{a}$ Época (1987-1993) que, tras diez volúmenes, pasaría a denominarse Demófilo. Revista de Cultura Tradicional de Andalucía, ya en la década de los 90. Con el cambio de dirección en 2000, tras un impasse de dos años, cambia de contenidos e inicia una tercera época (2002-2008), muy enfocada a los estudios de flamenco. También en Andalucía, la Consejería de Cultura de la Junta de Andalucía inicia en 1988 una publicación periódica, aunque con bastante irregularidad en los últimos años, a la que denomina Anuario Etnológico de Antropología, con el fin de recoger las investigaciones etnográficas subvencionadas por esta institución.

En Tenerife, en la Universidad de La Laguna, el Laboratorio de Antropología Social publica durante un tiempo el Boletín de Historia de la Antropología (1988-1989), coordinado por Fernando Estévez. También en 1988, desde el Ourensan de Antropoloxia Social, se publica Cadernos do Laboratorio Ourensan de Antropoloxia, coordinado por X.A. Fidalgo y X. Simal.

Finalmente, ya en 1989, por iniciativa de las profesoras del Departamento de Historia Contemporánea de la Universidad de Barcelona, Mercedes Vilanova y Cristina Borderías, aparece la revista Historia $y$ Fuente Oral, que a partir de 1996 pasaría de denominarse Historia, Antropología y Fuentes Orales, siendo editada por el Seminario de Historia Oral del Departamento de Historia Contemporánea de la Universidad de Barcelona, Arxiu Històric de la Ciutat de Barcelona y la Universidad de Granada, manteniéndose con periodicidad hasta el 2011.

En la década de los noventa podemos señalar una evidente disparidad geográfica entre las revistas antropológicas editadas en las diferentes Comunidades Autónomas, siendo Cataluña, Madrid, Andalucía y el País Vasco las que mayor número de publicaciones concentran. Cabe señalar que hubo un buen número de revistas que tuvieron una duración mínima, como Anuario de Historia de la Antropología Española (1992-1993) del Instituto de Antropología de Barcelona, o la Revista de Antropología Aplicada (1996) de la Sociedad Española de Antropología Aplicada, dirigida por Ma Jesús Buxóy Ángel Aguirre.

Y otras publicaciones entran con fuerza en el panorama estatal, unas con más éxito que otras. Antropología. Revista de Pensamiento Antropológico y Estudios Etnográficos (1991-1998), Ilegaría a publicar catorce números bajo la iniciativa del denominado Grupo Antropología, coordinada por A. Álvarez Roldán, A. Barañano, A. Díaz de Rada. Cultures. Revista Asturiana de Cultura (1991-2010), auspiciada por la Academia de la Llingua Asturiana, con una periodicidad anual ha publicado hasta dieciséis números. También en el año 1991, se inicia la Revista de Antropología Social (1991-) del 
Departamento de Antropología Social de la Universidad Complutense de Madrid, que se mantiene hasta la actualidad con periodicidad anual y con un total de veintiún números publicados.

En el año 1992, aparecen dos revistas vinculadas a dos instituciones no universitarias, en dos enclaves territoriales distintos. En Andalucía, el Centro de Investigaciones Etnológicas Ángel Ganivet, de Diputación Provincial de Granada, iniciaba la publicación de Fundamentos de Antropología (1992-2001), dirigida por José Antonio González Alcantud, que tras once números dejó de publicarse. Por el contrario, con una vigencia mantenida hasta la actualidad, en Cataluña aparece la Revista d'Etnología de Catalunya, del Centro de Promoción de la Cultura Popular y Tradicional Catalana (Departamento de Cultura de la Generalitat de Catalunya, Barcelona), que continúa hasta hoy habiéndose publicado en el 2012 el número treinta y ocho. En Canarias, desparecía la revista Eres casi a la par que se iniciaba la revista Guize (1994), gracias al impulso de la Asociación Canaria de Antropología y el Departamento de Prehistoria, Antropología e Historia Antigua, dirigida por José Alberto Galván. Asimismo, en esta fecha, desde la universidad de Murcia se publicaba la Revista Murciana de Antropología (1994-2008) que desaparecía con quince números. En ese mismo año de 1994 aparece Kobie. Antropología cultural, editada por la Diputación Foral de Navarra, que continúa hasta la actualidad, en 2011 había publicado quince volúmenes.

En 1997 aparecen dos revistas: Ankuregi. Revista de Antropología Social, de la Asociación Vasca de Antropología, actualmente en vigor, y Cuadernos de Antropología Social (Nueva Época) (1997), del Departamento de Antropología Social de la Universidad Hispalense, que no llegó a tener continuidad.

Durante estos años también encontramos publicaciones de carácter interdisciplinar en las que ocasionalmente se incluyen aportaciones antropológicas o cuyos contenidos son de un marcado carácter de etnografía regionalista que, por tanto, debemos señalar. Por ejemplo, en Galicia, los Cuadernos de Estudios Gallegoso los de la Institución Padre Sarmiento; en Extremadura, la revista Cuadernos Populares, en La Rioja, la revista del Instituto de Estudios Riojanos, en Baleares, la publicación del Institut d'Estudis Baleárics, en la Comunidad Valenciana, Arxius de Sociología, en Melilla, la revista El Vigía de Tierra, entre otras.

Con el nuevo siglo llegan las revistas electrónicas, que van a influir notablemente en la creación de nuevas publicaciones periódicas y en la trasformación de otras ya constituidas. De todas ellas la antropología española, y los antropólogos, saldrán enormemente reforzados. Citamos a continuación algunas revistas que se fundaron en años precedentes y que han adoptado el formato electrónico, simultaneándolo a veces con el formato impreso, siendo las más importantes: Revista de Dialectología y Tradiciones Populares, Revista Española de Antropología Americana, Cuadernos de Etnología y Etnografía de Navarra, Quaderns de l'Institut Catala d'Antropología, Gazeta de Antropología, Arxiu d'Etnografia de Catalunya. Revista d'Antropologia Social y Revista d'Etnología de Catalunya.

Como decimos, las revistas de antropología de más reciente creación tienden a presentarse en formato electrónico, sin por ello descuidar la calidad; todo lo contrario, tanto en su presentación como en los contenidos, siguiendo las normas y criterios internacionales para ser evaluadas e indexadas en las mejores bases de datos nacionales e internacionales.

En 2000 inicia su publicación una de las revistas españolas de mayor difusión en la actualidad, Aibr. Revista de Antropología Iberoamericana, de la Asociación de Antropólogos Iberoamericanos en RedAibr, publicando tanto en versión electrónica como impresa. Desde 2001 el Departamento de Antropología, Geografía e Historia de la Universidad de Jaén apoya la publicación de la Revista de Antropología Experimental, con una periodicidad anual, habiendo sacado a la luz ya trece números, dirigida por José Luis Anta Félez. Perifèria, nacida en 2004, surgió como una iniciativa de los/as doctorandos/as del Departamento de Antropología Social y Cultural de la Universitat Autònoma de Barcelona, apoyada por la dirección y el profesorado de su programa de Máster y Doctorado. Desde su inicio se ha centrado en difundir la experiencia y la producción investigadora de los/as doctorandos/as y estudiantes de Máster del Departamento, del tal modo que apoye su promoción, aprendizaje y trabajo colaborativo. (Con)textos: Revista d'antropologia i investigació social, nació en 2008 en el Departament 
d'Antropologia Cultural i Històriad'Amèrica i Àfrica de la Universitat de Barcelona, con cinco números editados hasta el momento de libre acceso. La Revista Valenciana d'Etnologia tuvo su aparición en 2006, patrocinada por el MuseuValencià d'Etnologia, se publica con periodicidad, tanto en formato digital con acceso libre a los textos como de forma impresa. ETNICEX. Revista de Estudios Etnográficos, surge en 2010 el seno de la Asociación Profesional Extremeña de Antropología (APEA) con la finalidad de recoger artículos, textos, documentos y noticias de antropología social, etnografía, patrimonio cultural, estudios de las culturas tradicionales y populares, referidos a Extremadura, España y Portugal, Europa e Iberoamérica; ya ha sacado a la calle 5 números, bajo la dirección de Javier Marcos Arévalo. En 2011 se ha publicado la Revista Andaluza de Antropología, auspiciada por la Asociación Andaluza de Antropología (ASANA), con vocación de acoger trabajos de profesionales de esta disciplina y de otras ciencias sociales, desde una pluralidad de marcos teóricos y metodologías, aunque privilegiando la perspectiva de las Antropologías del Sur, atentas sobre todo a las cuestiones claves de nuestro mundo contemporáneo y de las sociedades y sectores dependientes. Su periodicidad es semestral.

Con mirada amplia e histórica podemos decir que estas son las revistas que se han creado en las últimas décadas y que pueden ser consideradas como revistas con contenidos generales de antropología social y cultural. Desde luego, podríamos abrir el abanico e incluir otras que tienen secciones de antropología o abarcan un aspecto específico de ella. Esto supondría dilatar enormemente la cantidad de revistas, con el peligro de omitir títulos significativos. Sin embargo, no queremos dejar de nombrar las siguientes: Pasos. Revista de Turismo y Patrimonio Cultural, como una de nuestras revistas mejor situada y valorada, patrocinada por la Universidad de La Laguna, Revista de Folklore (gracias a la Fundación Joaquín Díaz), ETNIA-E: Cuadernos de investigación etnográfica sobre infancia, adolescencia y educación (dependiente del IMA/FMEE), Mus-A, TRANS, Revista Transcultural de Música, Boletín PH, etc.

A pesar de las dificultades para mantener a flote una publicación periódica, por los motivos señalados, observamos que cada vez hay más concienciación de lo que supone la existencia de publicaciones periódicas de calidad para la consolidación de la investigación antropológica en España. La producción científica desde la antropología depende, en gran parte, de su difusión y que esta se realice a través de los medios adecuados y de calidad. En este sentido, creemos que Gazeta de Antropología, tras treinta años contribuyendo al conocimiento de la antropología iberoamericana, es fundamental que continúe su andadura, hoy día ya adaptada a los nuevos tiempos y exigencias. Veamos su desarrollo temporal y temático con algún detenimiento.

\section{Gazeta de Antropología, un referente}

Como hemos indicado, Gazeta de Antropología nace junto a otras revistas en el territorio español, hace ahora 30 años. Entonces un grupo de personas -solo una estaba vinculada como profesor a la Universidad de Granada- decidió formar la Asociación Granadina de Antropología; a todos les unían fuertes lazos de amistad y, sobre todo, un interés especial por la Antropología Social. Pensaron que sería bueno tener un boletín de la asociación para, de manera especial, lograr comunicarse y difundir sus investigaciones y trabajos; serviría, al mismo tiempo, para estrechar relaciones con asociaciones afines y el intercambio con colegas y antropólogos de otras latitudes, no solo de España. Corría octubre de 1982 y en nuestro país los estudios de Antropología estaban reservados a un par de universidades, en la de Granada ni soñarlo. De aquí el enorme valor que tuvo dar forma a una asociación que se preocupara por las investigaciones etnográficas, las reflexiones antropológicas teóricas o los estudios locales y las tradiciones, entre otras; su gran aporte y valor fue proyectarlo desde fuera de las aulas universitarias o las instituciones académicas, más propensas a ello. Este nebuloso horizonte "se nutría de viejas militancias políticas, religiosas y ácratas, mutadas en acción cultural. Siempre en torno a pasiones", dice el editorial del número 10, celebrando el décimo aniversario. Muchos sabemos que editar una revista en papel es una empresa muy costosa, en lo personal y lo económico, ahora y siempre, luego tuvo mayor mérito sacarla a la luz sin el respaldo de una Universidad u otra institución. En las páginas anteriores, hemos visto cuántas revistas dejaron de publicarse por problemas de solvencia económica. 
Los fundadores de la Asociación Granadina de Antropología y Gazeta de Antropología ("con ' $\mathrm{z}$ ' de resistir") se propusieron contribuir al conocimiento científico antropológico -y filosófico en ocasiones- con un número anual. Puede parecer una periodicidad amplia, pero, como decimos, no era fácil, al no contar con subvención alguna (hubo incluso organismos públicos que comprometieron subvenciones que nunca se hicieron efectivas, salvo destacadas ocasiones, números 6, 8 y alguno más). Cierto es, el precio de su independencia le ha privado del favoritismo de los organismos oficiales y le ha obligado a sortear enormes dificultades para sufragarse, año a año. Pero solo en tres ocasiones ha faltado a la cita (1986, 1989 y 1994). Nada debe extrañarnos que en el editorial del número 12, correspondiente al año 1996, pueda leerse: "Los números publicados han ido saliendo adelante a base de dedicación voluntaria y voluntarista, de tenacidad y de ilusión de un grupo reducido de personas para pedir colaboraciones de artículos, pordiosear financiación y llevar a cabo nosotros mismos todas las tareas de edición y distribución".

Pero el paso de los años no mermó ni la vocación ni las ganas de continuar, intactas como al principio. Los impulsores de la Asociación y de Gazeta mantuvieron el proyecto en una situación de marginalidad y desconocimiento inicial de la disciplina por parte de las instituciones, pero "la semilla no fue vana ni cayó en terreno baldío. (...) Fue un oasis en el desierto", continúa el mismo editorial. Bajo su sombra funcionó un seminario de antropología, grupos de investigación, algún congreso y diferentes ciclos de conferencias que dieron la oportunidad de oír y ver entre los granadinos a algunos antropólogos de otras regiones de España y del extranjero. Quince años más tarde la licenciatura de Antropología Social y Cultural era un hecho en la Universidad de Granada. Estamos seguros de la contribución de aquellos fundadores para que esto sucediera, pues para entonces la práctica totalidad de ellos ya ejercían docencia en sus aulas, en titulaciones como Filosofía, Historia, Sociología o Trabajo Social. Desde entonces, profesores y estudiantes de doctorado han encontrado en Gazeta de Antropología un espacio extraordinario para dar a conocer sus investigaciones.

Cuando la revista cumplió su veinte aniversario (en 2002) José Luis Solana, nombrado director recientemente, recordaba en el editorial del número 18 y en un artículo en RDTP la andadura realizada, desde el papel a la web, esto es, desde su diseño artesanal y artículos escritos con máquina electrónica también revistas como Critique of Anthropology comenzaron a publicarse a multicopista- hasta la maquetación profesional del formato digital y cibernético, pasando por la imprenta. Todo ello, siempre, a costa del tiempo y saber hacer de su director, el profesor Pedro Gómez García, que ya sumaba entre los fundadores. A él se debe la feliz idea de llevarla a la red, cuando apenas había revistas alojadas en el ciberespacio; no se olvide que estamos hablando de 1998. Una vez más, Pedro Gómez ideó, impulsó y concretó el proyecto, diseñó la página web y asumió su mantenimiento y actualización. No sólo la hizo más atractiva, sino que la convirtió en una publicación de rango universal, al poder ser consultada por cualquier persona, desde no importa el ámbito geográfico del planeta. El editorial del número 14 (1998), anunciando el cambio, se felicitaba con estas palabras: "El que, por la versatilidad del medio, una revista tan modesta como Gazeta de Antropología pueda brindarse a todos los interesados con tanta eficacia es una tentación irrenunciable. Al fin hemos encontrado el mecenas que nunca llegamos a tener. En adelante todo dependerá sólo de la calidad de los autores y sus trabajos". Y ahí continúa. Esta posibilidad de consulta en abierto ha permitido que, según las estadísticas del contador Nedstat (incorporado a la página), para finales de 2012 Gazeta de Antropología haya recibido casi tres millones y medio de visitas (unas setecientas diarias); más de la mitad se realizan desde fuera de España, con presencia notable de 60 países (destacando México, Argentina, Estados Unidos, Chile, Colombia, Perú, Brasil, Portugal y Francia). Un buen refrendo a su calidad por parte de lectores e investigadores. Con el tiempo, y como no podía ser de otra manera, también han ido creciendo el Consejo de Dirección y el Comité Científico, cada vez más diversos e internacionales, muestra del apoyo y reconocimiento que recibe, así como el equipo de Dirección.

Los datos, aunque parezcan fríos, nos muestran una imagen bastante aproximada de la evolución de Gazeta en estas últimas tres décadas. Sandra Gómez Rivas los ha extraído de entre sus miles de páginas. Veamos respecto a los autores.

Han contribuido con sus trabajos 466 personas, de los que hay que tener en cuenta que una veintena 
solo ha colaborado escribiendo recensiones de libros (donde destaca Juan Javier Rivera). En total un elenco muy respetable. Como suele ser habitual, los autores que más publican en una revista son quienes mayor compromiso tienen con ella, por tanto, Demetrio Brisset (con 25 artículos), Pedro Gómez (con 24), José Luis Solana (con 20 publicaciones, entre artículos, traducciones y recensiones), J. Antonio González Alcantud (con 14 publicaciones) y Rafael Briones, Francisco Checa, Ángel Acuña o José Luis Anta (con una decena de artículos), son quienes mayor número de páginas aportan de su autoría. Cabe destacar también a antropólogos y filósofos, como Jesús J. Nebreda, Juan F. Gamella o Ester Massó y otros que, desde Latinoamérica, envían sus originales, como Luis Fernando Botero. Al margen de estos, Gazeta tiene entre sus números artículos de antropólogos tan nombrados como Claude Lévi-Strauss, Edgar Morin, Julio Caro Baroja o Julio Alvar; y entrevistas a personajes tan destacados como Martine Segalen (Centre d'Ethnologie Française, CNRS, París) o Rodolfo Sandoval (Secretario General del Consejo Supremo Purépecha, México). Ello no es óbice, bueno es reconocerlo, para admitir que, especialmente en las dos primeras décadas, muchos de los autores que se acercaban con sus trabajos a Gazeta de Antropología eran jóvenes investigadores -incluso estudiantes- con sus óperas primas, carentes de la calidad que otra publicación les exigiría; pero sus creadores la diseñaron para que también cupieran estos trabajos. El editorial del número 3 se manifiesta en este sentido: no están dispuestos a reducir la Antropología a una "carrera", solo a "una tarea de especialistas". Entienden la disciplina como una mirada hacia amplios horizontes "capaz de integrar tanto las descripciones de carácter empírico como las reflexiones de naturaleza teórica", por eso no solo se aceptan las reflexiones de especialistas consagrados, también los artículos etnográficos que llegan de jóvenes investigadores.

En una cifra tan elevada de autores lógicamente aparecen representadas todas las comunidades autónomas españolas -y sus universidades- y diferentes países europeos y latinoamericanos. Teniendo en cuenta desde donde se envían los originales, 103 autores residen en Andalucía, la mayor parte en Granada (59), Sevilla y Málaga (14); 138 trabajan o residen en ciudades españolas, la mayor parte de ellos en Madrid (36), Comunidad Valenciana (21), Murcia (18), Cataluña (17) y Extremadura (12). Otros 15 autores son europeos, desde Francia escriben 9 de ellos. El otro foco de gran relevancia está en Latinoamérica, pues nada menos que 142 autores mandan sus trabajos desde México (43), Argentina (37), Chile y Venezuela (17) o Perú (10), como más destacados.

El conjunto de artículos supera cualquier previsión inicial: 648 en estos treinta años. Si dividimos su aparición según la periodicidad de la revista, es decir, los primeros 23 volúmenes -anuales, entre 1982 y 2007- de los 10 restantes, semestrales, la distribución es la siguiente: 416 para los primeros y 230 para los segundos. Tiene mucho que ver la introducción de evaluadores en los últimos años, donde la selección es mayor y la exigencia de calidad una evidencia. De la decena de artículos de los primeros quince años, se pasó a la veintena en los años siguientes, incluso más de treinta artículos salieron a la luz en bastantes números. En la actualidad se vienen aceptando unos veinte estudios por número, y suele rechazarse sobre el cincuenta por ciento de los originales que llegan a la redacción. Por otro lado, a lo largo de estas tres décadas, Gazeta ha recensionado 106 obras, un promedio muy aceptable, teniendo en cuenta que hasta el número 7 no apareció la primera y que incluso en tres posteriores tampoco ha salido ninguna. Presentar a la comunidad científica libros recientemente publicados es muy de agradecer a quienes hacen el esfuerzo de leerlos con prontitud y sintetizarlos de manera crítica.

Las temáticas tratadas en Gazeta de Antropología han sido muy heterogéneas. Como ya destacaba José Luis Solana hace 10 años, existe un significativo número de contribuciones que se han ocupado de cuestiones de religiosidad popular, en su sentido más amplio, y de fiestas, tanto profanas como religiosas, donde destaca Demetrio Brisset: solo sobre las fiestas de Granada ha sacado a la luz hasta ocho artículos. En segundo lugar, podríamos constituir un extenso bloque compuesto con artículos sobre amplias temáticas que entrarían dentro de estudios etnográficos locales (la mayoría de pueblos andaluces), textos de claro carácter filosófico, sobre teoría antropológica y antropología fundamental, y contribuciones de temática americanista e indigenista. Más allá de estos conjuntos temáticos generales, un tercer apartado tendría tal variedad que abordada cuestiones tan diversas como las relacionadas con expresiones artísticas y literarias (música, artes plásticas, literatura, cine), la problemática de la identidad cultural, la marginación social y la pobreza, las desigualdades sociales, los gitanos (incluso los llegados de Rumanía), y artículos que entrarían dentro de las subdisciplinas de la antropología urbana, industrial o 
médica, la antropogerontología, la antropología jurídica o antropología visual; también sobre métodos y técnicas de investigación, la sexualidad, drogas, inmigración y globalización, incluso artículos sobre Port Aventura o el orgasmo femenino. En todos estos años sólo ha aparecido un monográfico, el dedicado a la arquitectura tradicional $(2000,16)$. Es muy probable que Gazeta de Antropología adolezca de la presentación de estudios monográficos, al estilo de otras, como por ejemplo hizo Demófilo sobre las provincias andaluzas, pero también sabemos que es una intención clara del nuevo equipo de Dirección y su Consejo de Redacción ir sacando cada año al menos uno ellos, dedicado a los temas de mayor actualidad.

Por otro lado, esta diversidad temática, como el editorial del número 10 señalaba, ha podido ser un hándicap en el conjunto de Gazeta: "uno de los grandes problemas aún no superados" de la revista es "la ausencia de puntos comunes de debate"; y añade:"ni siquiera la religiosidad popular, la temática más presente en los primeros diez números, los ha suscitado". En nuestra opinión, esa falta de puntos comunes, si ciertamente confiere un carácter heteróclito y quizá excesivamente disperso a la revista, desde otra perspectiva, sin embargo, ha posibilitado algunas de sus virtudes: la variedad de temáticas abordadas en los artículos, la apertura, esa sensación de plena libertad y de carencia de censuras o cortapisas que tanto los autores como los directores han sentido, y de la que siguen gozando quienes publican en ella.

No es, pues, la Gazeta una publicación temáticamente especializada. Pero debajo de sus variopintos contenidos creemos que pueden descubrirse talantes generales o vectores derivados, sobre todo, de los intereses y las relaciones personales e intelectuales de quienes, de un modo u otro, han contribuido -y están contribuyendo- a su progresivo desarrollo; líneas de orientación implícitas, nunca establecidas de modo explícito ni mucho menos impuestas.

En el editorial del número 13 -número en el que se introdujo una pequeña mejora, hoy imprescindible, los resúmenes de cada artículo en español e inglés- se abogaba por actualizar los "objetos" de estudio de la Antropología (algo que ha ido plasmándose en las publicaciones de esta revista), lo que en modo alguno debía entenderse como un rechazo o menosprecio hacia las temáticas y los campos de investigación antropológicos considerados como tradicionales, a los que la Gazeta siempre ha procurado prestar acogida, sino como una reivindicación de la necesidad, por los fructíferos resultados que vienen obteniéndose, de que la antropología persista y profundice en el estudio de los fenómenos propios de las sociedades complejas. "Las investigaciones sobre los temas tradicionales [decía el editorial] deben continuar, entre otras muchas razones porque el giro hacia una antropología de los mundos contemporáneos nunca podrá prescindir del estudio de esos otros mundos tradicionalmente estudiados por los antropólogos, pues, como magistralmente mostró Georges Balandier, para el estudio de la modernidad el desvío antropológico constituye un camino fecundo que llega a ser imprescindible".

Por lo demás, desde la Gazeta se ha intentado potenciar el análisis crítico-emancipatorio, orientado por ideales filantrópicos y procurando siempre que la crítica fuese juiciosa y fundamentada. A este respecto, en el número 13 (1997) se declaraba algo que aún guarda rabiosa vigencia: "En tiempos de estupidización mediática, de pérdida de referentes teóricos sólidos, críticamente fundados -que no dogmáticamente asumidos- de adormecimiento de la consciencia, de triunfos del capital, de desmonte en un pispás 'democrático' (tiempos, sí, también, de pudrimiento de las grandes palabras) de conquistas sociales arrancadas con sangre tras luchas seculares, de retorno de formas de explotación laboral que parecían extinguidas..., en estos ensombrecidos tiempos finiseculares, la Antropología debe asumir de modo radical un habermasiano interés crítico-emancipatorio". En esta línea, en el número 11 comenzaron a aparecer contribuciones sobre antropología compleja, resultado del estudio de la obra de Edgar Morin que algunos miembros de la revista, junto con sus doctorandos, venían desarrollando.

\section{Perspectivas actuales y de futuro}

Octubre de 1998, número 14 de la Gazeta de Antropología: omega y alfa. Número fronterizo que marca 
el paso desde el papel a la Red; número híbrido, pues fue publicado en papel, pero ampliado con contenidos -artículos y reseñas- nuevos en Internet. Desde entonces su acceso es libre y gratuito. La dirección web ha cambiado varias veces, esta se presume será quizá la definitiva: http://www.gazetaantropologia.es. Gana, desde entonces, que para leer un artículo no haya que esperar al fin del año o semestre, dado que la publicación es procesual: los artículos aparecen conforme van llegando y obtienen una evaluación positiva de los evaluadores externos.

Las ventajas de una edición de este tipo son muchas: es más económico, el número de páginas por número puede ser, si se quiere, prácticamente ilimitado, permite colgar la cantidad de fotografías, cuadros o gráficas que se deseen, incluso en color, se pueden ampliar los números de cada año gracias a los monográficos, como adelantamos, se goza de difusión internacional, etc. Hace un tiempo, se le otorgó el certificado de calidad doble U.com "por cumplir con excelentes estándares de calidad en su contenido, diseño, originalidad y claridad en la presentación de la página". El sello de calidad del FECYT, como "Excelente", respalda el trabajo realizado; la ANEP la cataloga con una categoría "A". Su incorporación en la base de datos de SciVerse-SCOPUS también es una buena noticia. Hace varios años que Gazeta de Antropología cumple los 36 criterios que Latindex establece para revistas electrónicas. Aparece en la base de datos de una quincena de catálogos, nacionales e internacionales; entre las revistas españolas de antropología (IN-RECS) lleva años instalada en el primer cuartil. Está considerada, a día de hoy, según estimación de la web especializada El Rincón del Antropólogo (http://www.elrincondelantropologo.com) como uno de los diez mejores sitios de antropología iberoamericana en Internet. $\mathrm{Y}$ en Internet se encuentran enlaces permanentes hacia la página de la Gazeta desde más de un centenar de páginas de importantes universidades, de países como Estados Unidos, Inglaterra, Francia, Suiza, Italia y de América Latina. Es para estar satisfechos por lo conseguido.

No obstante, como las expectativas de crecimiento de Gazeta siguen siendo excelentes, y de mejora, conviene considerar insuficiente lo alcanzado y continuar con el reto para la próxima década.

Para la celebración de este 30 Aniversario, la Junta de Dirección de Gazeta y nosotros como coordinadores del monográfico, queremos agradecer encarecidamente a todos los autores y autoras la colaboración. Ha sido un lujo contar con tan prestigiosas firmas. Desde el principio fuimos conscientes de que otros/as investigadores quedarían fuera, por una única razón: era imposible dar cabida a todas aquellas personas con las que deseábamos contar. Al menos hemos procurado que estén representados varios continentes, las Comunidades Autónomas, la mayoría de las universidades españolas, diferentes orientaciones antropológicas y distintas generaciones. Dentro de diez años prometemos completar el listado. A todos, gracias.

\section{Bibliografía}

Calvo Calvo, Luis (ed.)

2002 "Memoria del presente: la Antropología española en sus publicaciones periódicas", RDTP, LVII, 1.

Medina, F. Xavier ( y Ricardo Sánchez)

2002 "Diez años de revistas antropológicas en España (1990-2000): una evaluación”, RDTP, LVII, 1: 1128.

Prat i Caros, Joan

1990-91 "Les revues d'antrhropologie en Espagne", La Revue des renues, nº 10: 49-51.

Prat i Caros, Joan (coord.)

1999 “Investigadores e investigados. Literatura antropológica en España desde 1954", Arxiu d'Etnografia 
de Catalunya (edición especial).

Prat i Caros, Joan (dir./coord.)

2004 Las Ciencias Sociales en España: Historia inmediata, crítica y perspectivas. Vol. 2, Antropología y Etnología. Madrid, Editorial Complutense.

Sánchez Montañés, Emma (y María Josefa Iglesias)

2002 "La visión del otro. Breve historia de la Revista Española de Antropología Americana", RDTP, LVII, 1: $59-70$.

Solana Ruiz, José Luis

2002 "La Gazeta de Antropología: de la precariedad local al ciberespacio global”, RDTP, 57-1. 